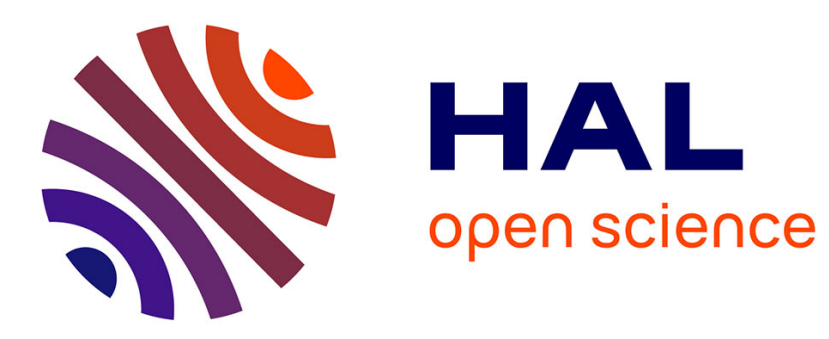

\title{
Efficient Pseudolinear Estimation for Bearings and Frequencies Target Motion Analysis
}

Claude Jauffret, Annie-Claude Perez

\section{To cite this version:}

Claude Jauffret, Annie-Claude Perez. Efficient Pseudolinear Estimation for Bearings and Frequencies Target Motion Analysis. International Conference on Information Fusion, Jul 2018, cambridge, United Kingdom. hal-01863573

\section{HAL Id: hal-01863573 https://hal.science/hal-01863573}

Submitted on 22 Sep 2021

HAL is a multi-disciplinary open access archive for the deposit and dissemination of scientific research documents, whether they are published or not. The documents may come from teaching and research institutions in France or abroad, or from public or private research centers.
L'archive ouverte pluridisciplinaire HAL, est destinée au dépôt et à la diffusion de documents scientifiques de niveau recherche, publiés ou non, émanant des établissements d'enseignement et de recherche français ou étrangers, des laboratoires publics ou privés. 


\title{
Efficient Pseudolinear Estimation for Bearings and Frequencies Target Motion Analysis
}

\author{
Claude Jauffret, Annie-Claude Pérez \\ Université de Toulon, Aix Marseille Univ, CNRS, IM2NP, Toulon, France \\ CS 60584, 83041 TOULON Cedex 9, France \\ annie-claude.perez@univ-tln.fr, jauffret@univ-tln.fr
}

\begin{abstract}
In this paper, we present a pseudolinear estimate for bearings and frequencies target motion analysis. We propose two ways to reduce its inherent bias and evaluate its performance with Monte Carlo simulations, with reference to the Cramér-Rao lower bound.
\end{abstract}

Keywords-bearings and frequencies target motion analysis, observability, maximum likelihood estimate, pseudolinear estimate, Cramér-Rao lower bound, bias reduction.

\section{INTRODUCTION}

Target motion analysis (TMA) is now an old problem wellknown by our community. The most common variants are probably bearings-only TMA (BOTMA) [1], and bearings and frequency (or Doppler) TMA (BFTMA) [2]. The first needs the platform to maneuver properly, whereas the second has no specific requirement to make the task feasible (observability condition). The solutions proposed in the literature can be classified into two main groups: the batch methods devoted to the scenarios during which the source does not maneuver, and those devoted to possibly maneuvering sources. For the first situation, the maximum likelihood estimate is most often proposed, computed via a numerical routine [1]. For the second situation, a recursive (compatible with real-time) filter is most appropriate: extended and unscented Kalman filters (with convenient coordinate systems) can be run, but with a risk of divergence of the filter [3] [4], particle filters with low risk of divergence but demanding a strong computational resource [5]. All these methods are iterative (or time-recursive, which is a kind of iteration). In this paper, we give the proof of observability in BFTMA in discrete time, the analysis of observability having been made in continuous time in the past [6]. Then, we propose two ways to compute directly two almost unbiased estimates in BFTMA by a straightforward computation. This is based upon the so-called modified instrumental method, initially introduced in [7]

The paper is organized as follows:

In section II, the problem formulation and our notations are presented. Section III is devoted to observability analysis in discrete time. We present in section IV a pseudolinear estimate, which is biased. Section V offers two ways to reduce the bias, and presents some simulations, illustrated with typical scenarios. A conclusion and an appendix, containing some computational details, follow.

\section{PROBLEM FORMULATION AND NOTATIONS}

We consider the common situation where a motionless observer acquires sequentially a couple of measurements composed of an azimuth (or bearing) and a frequency of a single tone emitted by a source moving at constant velocity. Due to the source's motion, the detected frequency is Dopplershifted. The plane in which the two actors play is given a Cartesian coordinate system. In this system, the position vector at time $t$ and the velocity vector of the source are $P_{S}(t)=\left[\begin{array}{ll}x_{S}(t) & y_{S}(t)\end{array}\right]^{T}$ and $V_{S}=\left[\begin{array}{ll}\dot{x}_{S} & \dot{y}_{S}\end{array}\right]^{T}$, respectively. The vectors $P_{S}(t)$ and $V_{S}$ are also described by their polar coordinates: $\quad P_{S}(t)=r(t)[\sin \theta(t) \cos \theta(t)]^{T} \quad$ and $V_{S}=v\left[\begin{array}{ll}\sin h & \cos h\end{array}\right]^{T}$. The source is assumed to be emitting a pure single tone at the unknown frequency $\gamma_{0}$. The observer is located at $P_{O}=\left[\begin{array}{ll}0 & 0\end{array}\right]^{T}$. At time $t_{k}$, the noiseless azimuth and frequency are respectively $\theta_{k}=\theta\left(t_{k}\right)=\operatorname{Atan}\left[x_{S}\left(t_{k}\right), y_{S}\left(t_{k}\right)\right]$ and $f_{k}=\gamma_{0}\left[1-\frac{\dot{r}_{k}}{c}\right]=\gamma_{0}\left[1-\frac{\dot{x}_{S} \sin \theta_{k}+\dot{y}_{S} \cos \theta_{k}}{c}\right]$, where $\dot{r}_{k}$ stands for $\frac{\mathrm{d} r}{\mathrm{dt}}\left(t_{k}\right)$. Note that in these two expressions the propagation delay is neglected. The letter c denotes the speed of sound in the medium $(\mathrm{c}=1500 \mathrm{~m} / \mathrm{s}$ in water). At this time, the observer acquires the pair of measurements $\left(\theta_{m, k}, f_{m, k}\right)$ defined by $\theta_{m, k}=\theta_{k}+\varepsilon_{k}$ and $f_{m, k}=f_{k}+\eta_{k}$. The terms $\varepsilon_{k}$ and $\eta_{k}$ are respectively the measurement noises corrupting the bearing and the frequency acquired at time $t_{k}$. The noise vector $\left.\left[\begin{array}{llllll}{\left[\varepsilon_{1}\right.} & \eta_{1}\end{array}\right]^{T} \quad \cdots \quad\left[\begin{array}{lll}\varepsilon_{k} & \eta_{k}\end{array}\right]^{T} \quad \cdots \quad\left[\begin{array}{ll}\varepsilon_{K} & \eta_{K}\end{array}\right]^{T}\right]^{T}$ is assumed to be a zero-mean Gaussian vector whose covariance matrix is diagonal. With no loss of generality, we will assume that $\operatorname{Var}\left(\varepsilon_{k}\right)$ and $\operatorname{Var}\left(\eta_{k}\right)$ are constant, i.e. $\operatorname{Var}\left(\varepsilon_{k}\right)=\sigma_{\theta}^{2}$ and $\operatorname{Var}\left(\eta_{k}\right)=\sigma_{f}^{2}$.

The purpose of BFTMA is to estimate the trajectory of the source from a set of collected measurements $\left\{\left(\theta_{m, k}, f_{m, k}\right), k=1, \ldots, K\right\}$. Because $\gamma_{0}$ is unknown, it must be estimated, as well.

The "natural" state vector that characterizes the source (i.e. its motion and its "music", that is $\gamma_{0}$ ) should be $\left[\begin{array}{lllll}x_{S}\left(t_{1}\right) & y_{S}\left(t_{1}\right) & \dot{x}_{S} & \dot{y}_{S} & \gamma_{0}\end{array}\right]^{T}$. However, we make another choice: the state vector defined as $X=\left[\begin{array}{lllll}\gamma_{0} x_{S}\left(t_{1}\right) & \gamma_{0} y_{S}\left(t_{1}\right) & \gamma_{0} \dot{x}_{S} & \gamma_{0} \dot{y}_{S} & \gamma_{0}\end{array}\right]^{T}$ is more convenient to 
establish the observability of the trajectory from the set of noiseless measurements $\left\{\left(\theta_{k}, f_{k}\right), k=1, \ldots, K\right\}$ whose proof is given in the next section, and to construct a pseudolinear estimate. This state vector was proposed first in [1], then later used in [2].

\section{OBSERVABILITY ANALYSIS IN DISCRETE TIME}

The analysis of the observability is conducted in discrete time. This is thus the counterpart of the proof in continuous time given in [6]. Before giving the main result, we recall the following property (the proof is omitted):

\section{Lemma}

The three statements are equivalent:

(i) The source's trajectory is aligned with the observer's position,

(ii) Two bearings are equal,

(iii) All the bearings are equal.

The conclusion about observability is summarized by the:

\section{Proposition}

If the source's trajectory is not aligned with the observer's position, then its trajectory is observable from three pairs of measurements $\left\{\left(\theta_{k}, f_{k}\right), k=1,2,3\right\}$.

Proof:

At time $t_{k}$, the noiseless measurements $\left(\theta_{k}, f_{k}\right)$ and the state vector satisfy the following linear system: $\left[\begin{array}{ccccc}\cos \theta_{k} & -\sin \theta_{k} & t_{k} \cos \theta_{k} & -t_{k} \sin \theta_{k} & 0 \\ 0 & 0 & -\frac{\sin \theta_{k}}{c} & -\frac{\cos \theta_{k}}{c} & 1\end{array}\right] X=\left[\begin{array}{c}0 \\ f_{k}\end{array}\right]$.

With three pairs of measurements $\left\{\left(\theta_{k}, f_{k}\right), k=1,2,3\right\}$, the state vector $X$ must satisfy the linear system (we implicitly assume that $t_{1}=0$ )

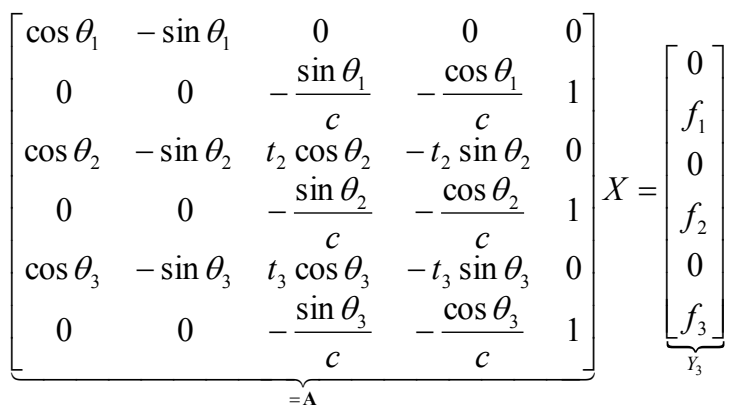

which is equivalent to

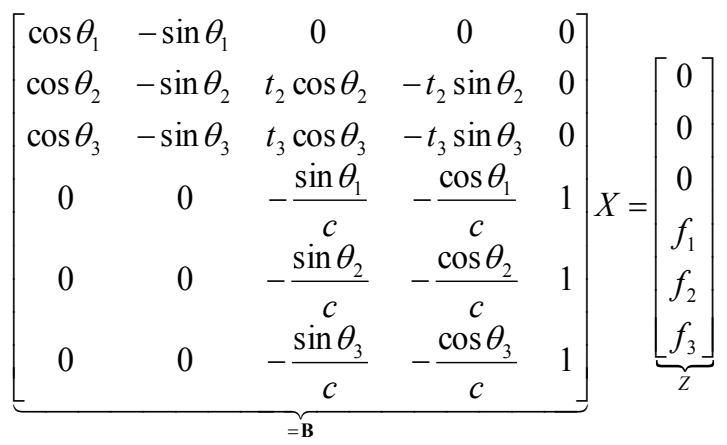

We are going to prove that $X$ is unique, i.e. if another vector $X^{\prime}$ satisfies the equation $\mathbf{B} X^{\prime}=Z$ then $X^{\prime}=X$ or equivalently, the kernel of $\mathbf{B}$ is the null vector of $R^{5}$.

First step:

Let us consider the submatrix $\left[\begin{array}{ccc}-\frac{\sin \theta_{1}}{c} & -\frac{\cos \theta_{1}}{c} & 1 \\ -\frac{\sin \theta_{2}}{c} & -\frac{\cos \theta_{2}}{c} & 1 \\ -\frac{\sin \theta_{3}}{c} & -\frac{\cos \theta_{3}}{c} & 1\end{array}\right]$.

Obviously, its determinant is proportional to the determinant of $\quad \mathbf{C}=\left[\begin{array}{lll}-\sin \theta_{1} & -\cos \theta_{1} & 1 \\ -\sin \theta_{2} & -\cos \theta_{2} & 1 \\ -\sin \theta_{3} & -\cos \theta_{3} & 1\end{array}\right]$ which is equal to $\operatorname{det}(\mathbf{C})=\sin \left(\theta_{1}-\theta_{2}\right)+\sin \left(\theta_{2}-\theta_{3}\right)+\sin \left(\theta_{3}-\theta_{1}\right)$.

We thus have $\operatorname{det}(\mathbf{C})=0 \Leftrightarrow \sin \left(\theta_{1}-\theta_{2}\right)+\sin \left(\theta_{2}-\theta_{3}\right)=\sin \left(\theta_{1}-\theta_{3}\right)$. $\Leftrightarrow 2 \sin \left(\frac{\theta_{1}-\theta_{3}}{2}\right) \cos \left(\frac{\theta_{1}+\theta_{3}}{2}-\theta_{2}\right)=2 \sin \left(\frac{\theta_{1}-\theta_{3}}{2}\right) \cos \left(\frac{\theta_{1}-\theta_{3}}{2}\right)$ $\Leftrightarrow \sin \left(\frac{\theta_{1}-\theta_{3}}{2}\right)\left[\cos \left(\frac{\theta_{1}-\theta_{3}}{2}\right)-\cos \left(\frac{\theta_{1}+\theta_{3}}{2}-\theta_{2}\right)\right]=0$ $\Leftrightarrow \sin \left(\frac{\theta_{1}-\theta_{3}}{2}\right) \sin \left(\frac{\theta_{1}-\theta_{2}}{2}\right) \sin \left(\frac{\theta_{2}-\theta_{3}}{2}\right)=0$

So $\operatorname{det}(C)=0 \Leftrightarrow$ one of the last three conditions is verified: $\theta_{1}=\theta_{3}(\bmod 2 \pi)$

$\theta_{1}=\theta_{2}(\bmod 2 \pi)$

$\theta_{2}=\theta_{3}(\bmod 2 \pi)$

The conclusion of this first step is that the matrix $\mathbf{C}$ is nonsingular since all the bearings are different; that is, the source's trajectory is not aligned with the observer's location.

\section{Second step:}

Let us identify the kernel of $\mathbf{B}$. We have to solve the equation $\mathbf{B} X=\left[\begin{array}{llllll}0 & 0 & 0 & 0 & 0 & 0\end{array}\right]^{T}$.

The $\mathrm{i}^{\text {th }}$ component of $X$ being denoted $x_{i}$, the last three rows of the above equation can be written as

$\mathbf{C}\left[\begin{array}{lll}x_{3} & x_{4} & x_{5}\end{array}\right]^{T}=\left[\begin{array}{lll}0 & 0 & 0\end{array}\right]^{T}$. Since $\mathbf{C}$ is nonsingular, this yields $\left[\begin{array}{lll}x_{3} & x_{4} & x_{5}\end{array}\right]^{T}=\left[\begin{array}{lll}0 & 0 & 0\end{array}\right]^{T}$.

Now reporting this result in the first two rows, we get the system

$$
\left[\begin{array}{cc}
\cos \theta_{1} & -\sin \theta_{1} \\
\cos \theta_{2} & -\sin \theta_{2} \\
\cos \theta_{3} & -\sin \theta_{3}
\end{array}\right]\left[\begin{array}{l}
x_{1} \\
x_{2}
\end{array}\right]=\left[\begin{array}{l}
0 \\
0 \\
0
\end{array}\right] \text {, whose solution is }\left[\begin{array}{l}
x_{1} \\
x_{2}
\end{array}\right]=\left[\begin{array}{l}
0 \\
0
\end{array}\right] \text {. }
$$

The conclusion of step 2 is that the kernel of $\mathbf{B}$ is the null vector of $R^{5}$. As a consequence, the equation $\mathbf{B} X^{\prime}=Z$ has a unique solution if and only if the source's trajectory is not aligned with the observer's position. QED.

In the sequel, we will assume that $t_{k}=(k-1) \Delta t$ and $\Delta t=\frac{T}{K-1}, T$ being the duration of the scenario.

\section{THE PSEUdolinear ESTIMATE}

The basic idea of the so-called "pseudolinear" estimate comes from the possibility of transforming a nonlinear measurement 
equation into a linear form, the price being that the noise is no longer additive. From our knowledge, the first paper invoking this transformation is [7], for BOTMA. Since then, this technique has been widely re-used for BFTMA as well [2, 9, $10]$.

\section{A. Construction of the estimate}

Suppose now that $K$ pairs of measurements are available. Let us apply the linear transformation that allowed us to get (1) to the data:

\section{Proposition}

$$
\left[\begin{array}{c}
0 \\
f_{m, k}
\end{array}\right]=\mathbf{M}_{k} X+\xi_{k}
$$

with

$\xi_{k}=-\gamma_{0}\left[\frac{v}{c}\left[\cos \left(\theta_{k}-h\right)\left(1-\cos \varepsilon_{k}\right)+\sin \left(\theta_{k}-h\right) \sin \varepsilon_{k}\right]\right]+\left[\begin{array}{c}0 \\ \eta_{k}\end{array}\right]$

and $\mathbf{M}_{k}=\left[\begin{array}{ccccc}\cos \theta_{m, k} & -\sin \theta_{m, k} & t_{k} \cos \theta_{m, k} & -t_{k} \sin \theta_{m, k} & 0 \\ 0 & 0 & -\frac{\sin \theta_{m, k}}{c} & -\frac{\cos \theta_{m, k}}{c} & 1\end{array}\right]$

where $r_{k}$ stands for $r\left(t_{k}\right)$.

Proof:

- The bearings:

We compute the expression $x_{k} \cos \theta_{m, k}-y_{k} \sin \theta_{m, k}$ (similar to $\left.x_{k} \cos \theta_{k}-y_{k} \sin \theta_{k}=0\right)$ :

$$
\begin{aligned}
x_{k} \cos \theta_{m, k}-y_{k} \sin \theta_{m, k} & =x_{k} \cos \left(\theta_{k}+\varepsilon_{k}\right)-y_{k} \sin \left(\theta_{k}+\varepsilon_{k}\right) \\
& =r_{k} \sin \theta_{k} \cos \left(\theta_{k}+\varepsilon_{k}\right)-r_{k} \cos \theta_{k} \sin \left(\theta_{k}+\varepsilon_{k}\right) \\
& =r_{k} \sin \left(\theta_{k}-\theta_{k}-\varepsilon_{k}\right) \\
& =-r_{k} \sin \varepsilon_{k} .
\end{aligned}
$$

That is,

$x_{k} \cos \theta_{m, k}-y_{k} \sin \theta_{m, k}=-r_{k} \sin \varepsilon_{k}$.

By using the state vector $X$, Eq. (2) becomes:

$\left[\begin{array}{lllll}\cos \theta_{m, k} & -\sin \theta_{m, k} & t_{k} \cos \theta_{m, k} & -t_{k} \sin \theta_{m, k} & 0\end{array}\right] X=-\gamma_{0} r_{k} \sin \varepsilon_{k}$.

- The frequency

Now, we compute $\dot{x} \sin \theta_{m, k}+\dot{y} \cos \theta_{m, k}$ :

$\dot{x} \sin \theta_{m, k}+\dot{y} \cos \theta_{m, k}=v \sin h \sin \theta_{m, k}+v \cos h \cos \theta_{m, k}$

$$
\begin{aligned}
& =v \cos \left(\theta_{m, k}-h\right) \\
& =v \cos \left(\theta_{k}-h\right) \cos \varepsilon_{k}-v \sin \left(\theta_{k}-h\right) \sin \varepsilon_{k}
\end{aligned}
$$

As a consequence,

$$
\begin{aligned}
& \gamma_{0}\left(1-\frac{\dot{x}}{c} \sin \theta_{m, k}-\frac{\dot{y}}{c} \cos \theta_{m, k}\right)= \\
& \gamma_{0}\left[1-\frac{v}{c} \cos \left(\theta_{k}-h\right) \cos \varepsilon_{k}+\frac{v}{c} \sin \left(\theta_{k}-h\right) \sin \varepsilon_{k}\right]= \\
& f_{k}+\gamma_{0} \frac{v}{c}\left[\cos \left(\theta_{k}-h\right)\left(1-\cos \varepsilon_{k}\right)+\sin \left(\theta_{k}-h\right) \sin \varepsilon_{k}\right]= \\
& f_{k, m}+\gamma_{0} \frac{v}{c}\left[\cos \left(\theta_{k}-h\right)\left(1-\cos \varepsilon_{k}\right)+\sin \left(\theta_{k}-h\right) \sin \varepsilon_{k}\right]-\eta_{k}
\end{aligned}
$$

We end up with the following pseudolinear measurement equation:

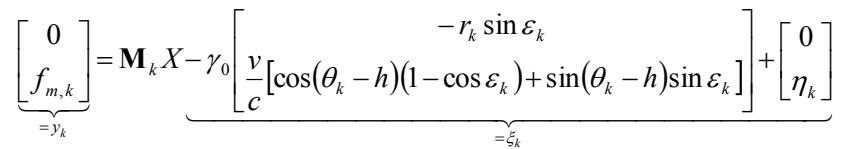

for $k=1, \cdots, K$.

QED.

Now, we construct the following pseudolinear estimate $\hat{X}=\left(\sum_{k=1}^{K} \mathbf{M}_{k}^{T} \mathbf{M}_{k}\right)^{-1}\left(\sum_{k=1}^{K} \mathbf{M}_{k}^{T} y_{k}\right)$, designated by PSLE.

Due to the poor conditioning of $\sum_{k=1}^{K} \mathbf{M}_{k}^{T} \mathbf{M}_{k}$, it is convenient ${ }^{1}$ to weight the pseudo measurements vector $y_{K}$ with the inverse of the covariance matrix $\mathbf{R}_{k}$ of the pseudo noise $\xi_{k}$ :

$$
\begin{aligned}
\hat{X} & =\left(\sum_{k=1}^{K} \mathbf{M}_{k}^{T} \mathbf{R}_{k}^{-1} \mathbf{M}_{k}\right)^{-1}\left(\sum_{k=1}^{K} \mathbf{M}_{k}^{T} \mathbf{R}_{k}^{-1} y_{k}\right) \\
& =X+\left(\sum_{k=1}^{K} \mathbf{M}_{k}^{T} \mathbf{R}_{k}^{-1} \mathbf{M}_{k}\right)^{-1}\left(\sum_{k=1}^{K} \mathbf{M}_{k}^{T} \mathbf{R}_{k}^{-1} \xi_{k}\right)
\end{aligned}
$$

We deduce that

$$
\mathbf{E}(\hat{X})=X+\mathbf{E}\left\{\left(\sum_{k=1}^{K} \mathbf{M}_{k}^{T} \mathbf{R}_{k}^{-1} \mathbf{M}_{k}\right)^{-1}\left(\sum_{k=1}^{K} \mathbf{M}_{k}^{T} \mathbf{R}_{k}^{-1} \xi_{k}\right)\right\} .
$$

Because of the presence of the noise in $\mathbf{M}_{k}$,

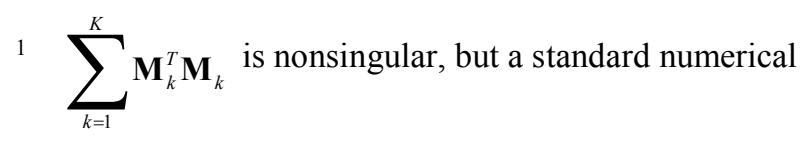
procedure of inversion can fail. 
$\mathbf{E}\left\{\left(\sum_{k=1}^{K} \mathbf{M}_{k}^{T} \mathbf{R}_{k}^{-1} \mathbf{M}_{k}\right)^{-1}\left(\sum_{k=1}^{K} \mathbf{M}_{k}^{T} \mathbf{R}_{k}^{-1} \xi_{k}\right)\right\}$ is non zero. This is the

bias of $\hat{X}$, denoted $\mathbf{b}$. We propose to approximate $\mathbf{R}_{k}$ as follows:

When $\sigma_{\theta}<<1$, then $\xi_{k} \cong \gamma_{0} \sin \varepsilon_{k}\left[\begin{array}{c}-r_{k} \\ \frac{v}{c} \sin \left(\theta_{k}-h\right)\end{array}\right]+\left[\begin{array}{c}0 \\ -\eta_{k}\end{array}\right]$ and its covariance matrix can be readily computed:

$\mathbf{R}_{k} \cong\left[\begin{array}{cc}\gamma_{0}^{2} r_{k}^{2} \sigma_{\theta}^{2} & -\gamma_{0}^{2} r_{k} \frac{v}{c} \sin \left(\theta_{k}-h\right) \sigma_{\theta}^{2} \\ -\gamma_{0}^{2} r_{k} \frac{v}{c} \sin \left(\theta_{k}-h\right) \sigma_{\theta}^{2} & \gamma_{0}^{2} \frac{v^{2}}{c^{2}} \sin ^{2}\left(\theta_{k}-h\right) \sigma_{\theta}^{2}+\sigma_{f}^{2}\end{array}\right]$
$\Rightarrow \mathbf{R}_{k}^{-1} \cong \frac{1}{\sigma_{f}^{2}}\left[\begin{array}{ccc}\frac{v^{2}}{c^{2} r_{k}^{2}} \sin ^{2}\left(\theta_{k}-h\right)+\frac{1}{\gamma_{0}^{2} r_{k}^{2}} \frac{\sigma_{f}^{2}}{\sigma_{\theta}^{2}} & \frac{v}{c r_{k}} \sin \left(\theta_{k}-h\right) \\ \frac{v}{c r_{k}} \sin \left(\theta_{k}-h\right) & 1\end{array}\right]$.

Note that the multiplicative term $\frac{1}{\sigma_{f}^{2}}$ does not intervene in the weighting. Three elements of $\mathbf{R}_{k}^{-1}$ are unknown. Fortunately, their orders of magnitude can be easily given: for a vessel or a submarine, the speed is about $10 \mathrm{~m} / \mathrm{s}$, and $\frac{\sigma_{f}}{\gamma_{0}} \approx 10^{-3}$; the range is between 5 and $20 \mathrm{~km}$ and $\sigma_{\theta}$ is close to $1^{\circ}\left(\frac{\pi}{180}\right.$ rad). These values allow us to approximate (even very roughly) $\mathbf{R}_{k}^{-1}$ by $\left[\begin{array}{cc}10^{-11} & 10^{-6} \\ 10^{-6} & 1\end{array}\right]=\Omega$. The elements of $\Omega$ are denoted $\omega_{i j}$.

\section{B. The problem of the bias}

Computing the bias analytically is hopeless: indeed, if such a computation was tractable, we would end up with an unbiased estimator; based on a fundamental theorem of mathematical statistics, this estimator would be the MLE and the model would be linear, which is not the case. The only achievable aim is to approximate or to estimate the bias $\mathbf{b}$.

In the open literature, two major ways to reduce this bias are proposed:

-The so-called modified instrumental variable estimate which consists in iterating the formula up to convergence

$$
\hat{X}_{p}=\left(\sum_{k=1}^{K} \hat{\mathbf{M}}_{k, p-1}^{T} \mathbf{R}_{k}^{-1} \mathbf{M}_{k}\right)^{-1}\left(\sum_{k=1}^{K} \hat{\mathbf{M}}_{k, p-1}^{T} \mathbf{R}_{k}^{-1} y_{k}\right),
$$

where $\hat{\mathbf{M}}_{k, p-1}$ is the matrix $\mathbf{M}_{k}$ constructed with the estimated bearings computed from $\hat{X}_{p-1}$. The reduction of the bias is justified by invoking Slutski's theorem [11].
- The estimation of the bias: once $\hat{\mathbf{b}}$ is obtained, we compute $\hat{X}-\hat{\mathbf{b}}$. This is our choice and we present two estimates in the next section.

\section{BIAS REDUCTION}

\section{A. Bias estimation: first solution}

To estimate the bias, we will employ the following trick: we artificially introduce the factor $K$ up and down in the expression of $\mathbf{b}$ :

$$
\mathbf{b}=\mathbf{E}\left\{\left(\frac{1}{K} \sum_{k=1}^{K} \mathbf{M}_{k}^{T} \mathbf{R}_{k}^{-1} \mathbf{M}_{k}\right)^{-1}\left(\frac{1}{K} \sum_{k=1}^{K} \mathbf{M}_{k}^{T} \mathbf{R}_{k}^{-1} \xi_{k}\right)\right\} .
$$

So, the two terms appear as an average of two quantities. As a consequence, $\mathbf{b}$ is approximated by $\mathbf{E}\left\{\left[\frac{1}{K} \sum_{k=1}^{K} \mathbf{M}_{k}^{T} \mathbf{\Omega} \mathbf{M}_{k}\right]^{-1}\right\}\left[\mathbf{E}\left[\frac{1}{K} \sum_{k=1}^{K} \mathbf{M}_{k}^{T} \mathbf{\Omega} \xi_{k}\right]\right.$ provided $K$ is large enough. The justification of this technique, pioneered by Dogancay [8], requires that the random matrix $\frac{1}{K} \sum_{k=1}^{K} \mathbf{M}_{k}^{T} \mathbf{\Omega} \mathbf{M}_{k}$ converges in distribution to a deterministic nonsingular matrix and that the random vector $\frac{1}{K} \sum_{k=1}^{K} \mathbf{M}_{k}^{T} \mathbf{\Omega} \xi_{k}$ converges in distribution to a deterministic vector.

This needs us to compute analytically $\mathbf{E}\left[\sum_{k=1}^{K} \mathbf{M}_{k}^{T} \mathbf{\Omega} \xi_{k}\right]$. A simple calculation ends up with

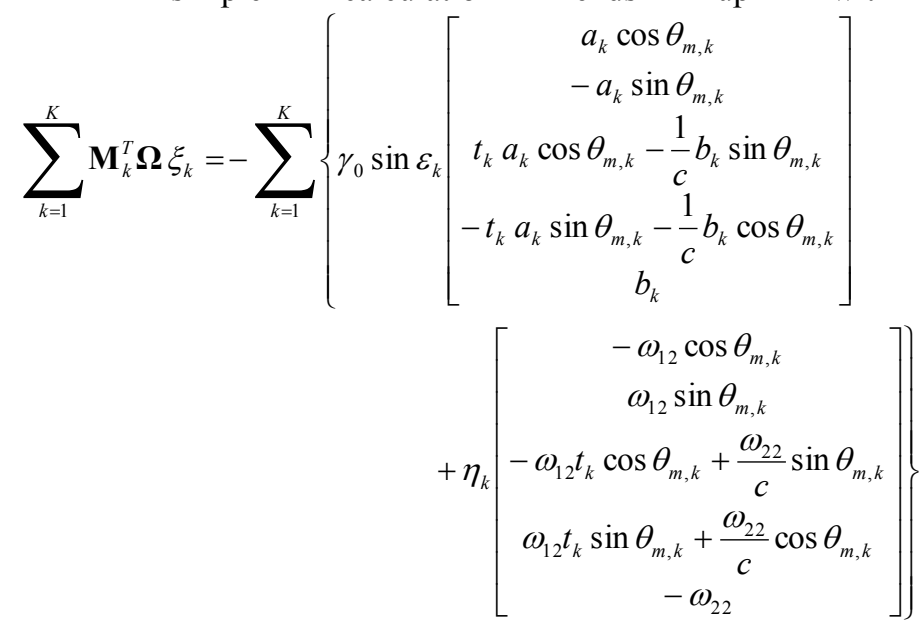

where

$$
a_{k}=-\omega_{11} r_{k}+\omega_{12} \frac{v}{c} \sin \left(\theta_{k}-h\right)
$$

and $b_{k}=-\omega_{12} r_{k}+\omega_{22} \frac{v}{c} \sin \left(\theta_{k}-h\right)$. Its mathematical expectation is 
$\mathbf{E}\left(\sum_{k=1}^{K} \mathbf{M}_{k}^{T} \mathbf{\Omega} \xi_{k}\right) \cong \sigma_{\theta}^{2} \gamma_{0}\left[\begin{array}{c}\sum_{k=1}^{K} a_{k} \sin \theta_{k} \\ \sum_{k=1}^{K} a_{k} \cos \theta_{k} a_{k} \sin \theta_{k}+\frac{1}{c} b_{k} \cos \theta_{k} \\ \sum_{k=1}^{K} t_{k} a_{k} \cos \theta_{k}-\frac{1}{c} b_{k} \sin \theta_{k} \\ 0\end{array}\right]$

since

$\varepsilon_{k}$ and $\eta_{k}$ are independent (see the lemma given in the Appendix).

In practice, its value is estimated according to the method of moments [12] by

$$
\begin{aligned}
& \sigma_{\theta}^{2} \hat{\gamma}_{0} \sum_{k=1}^{K}\left[\begin{array}{c}
\hat{a}_{k} \sin \theta_{m, k} \\
\hat{a}_{k} \cos \theta_{m, k} \\
t_{k} \hat{a}_{k} \sin \theta_{m, k}+\frac{1}{c} \hat{b}_{k} \cos \theta_{m, k} \\
t_{k} \hat{a}_{k} \cos \theta_{m, k}-\frac{1}{c} \hat{b}_{k} \sin \theta_{m, k} \\
0
\end{array}\right] \text { with } \\
& \hat{a}_{k}=-\omega_{11} \hat{r}_{k}+\omega_{12} \frac{\hat{v}}{c} \sin \left(\theta_{m, k}-\hat{h}\right) \text { and } \hat{b}_{k}=-\omega_{12} \hat{r}_{k}+\omega_{22} \frac{\hat{v}}{c} \sin \left(\theta_{m, k}-\hat{h}\right) \text {. }
\end{aligned}
$$

The computation of $\mathbf{E}\left[\frac{1}{K} \sum_{k=1}^{K} \mathbf{M}_{k}^{T} \mathbf{\Omega} \mathbf{M}_{k}\right]^{-1}$ being impossible, it is estimated by $\left[\frac{1}{K} \sum_{k=1}^{K} \mathbf{M}_{k}^{T} \Omega \mathbf{M}_{k}\right]^{-1}$. Finally we get an estimate $\hat{\mathbf{b}}$ of $\mathbf{b}$ and we replace $\hat{X}$ by $\hat{X}-\hat{\mathbf{b}}$. We call this an unbiased pseudolinear estimate (UPSLE).

\section{B. Bias estimation: second solution}

Another way to estimate the bias is to compute the value b for which the least squares criterion is the least:

$$
C(\mathbf{b})=\sum_{k=1}^{K} \frac{1}{\sigma_{\theta}^{2}}\left[\theta_{m, k}-\theta_{k}(\hat{X}-\mathbf{b})\right]^{2}+\frac{1}{\sigma_{f}^{2}}\left[f_{m, k}-f_{k}(\hat{X}-\mathbf{b})\right]^{2},
$$

approximated by

$$
\begin{gathered}
=\sum_{k=1}^{K} \frac{1}{\sigma_{\theta}^{2}}\left[\theta_{m, k}-\theta_{k}(\hat{X})+\nabla_{X}^{T} \theta_{k}(\hat{X}) \mathbf{b}\right]^{2} \\
+\frac{1}{\sigma_{f}^{2}}\left[f_{m, k}-f_{k}(\hat{X})+\nabla_{X}^{T} f_{k}(\hat{X}) \mathbf{b}\right]^{2}
\end{gathered}
$$

We end up with

$$
\begin{aligned}
\hat{\mathbf{b}}= & -\left[\sum_{k=1}^{K} \frac{1}{\sigma_{\theta}^{2}} \nabla_{X} \theta_{k}(\hat{X}) \nabla_{X}^{T} \theta_{k}(\hat{X})+\frac{1}{\sigma_{f}^{2}} \nabla_{X} f_{k}(\hat{X}) \nabla_{X}^{T} f_{k}(\hat{X})\right]^{-1} \\
& \sum_{k=1}^{K} \frac{1}{\sigma_{\theta}^{2}} \nabla_{X} \theta_{k}(\hat{X})\left[\theta_{m, k}-\theta_{k}(\hat{X})\right]+\frac{1}{\sigma_{f}^{2}} \nabla_{X} f_{k}(\hat{X})\left[f_{m, k}-f_{k}(\hat{X})\right] .
\end{aligned}
$$

In this expression, $\theta_{k}(X)$ and $f_{k}(X)$ are the mathematical bearing and frequency corresponding to a trajectory defined by $X$. We recognize the inverse of the Fisher information matrix (i.e. the CRLB) evaluated at $\hat{X}$. Again, we replace $\hat{X}$ by $\hat{X}-\hat{\mathbf{b}}$. Note that $\hat{X}-\hat{\mathbf{b}}$ is the approximate maximum likelihood estimate (AMLE).

We give in the next subsection some examples to evaluate the respective performance of each method for some scenarios.

\section{Examples}

In the two following examples, the observer is located at $\left[\begin{array}{ll}0 & 0\end{array}\right]^{T}$, the source is moving with a constant speed of $6 \mathrm{~m} / \mathrm{s}$ and a heading equal to $90^{\circ}$. Its initial position is $\left[\begin{array}{ll}0 & 9000\end{array}\right]^{T}(\mathrm{~m})$ in the first example and $\left[\begin{array}{ll}-3576 & 9000\end{array}\right]^{T}(\mathrm{~m})$ in the second one. The number of measurements is 150 , with a sampling period $\Delta t$ equal to $4 \mathrm{~s}$; the standard deviations are $\sigma_{\theta}=0.5^{\circ}$ and $\sigma_{f}=0.5 \mathrm{~Hz}$, while $\gamma_{0}=500 \mathrm{~Hz}$.

The performance of the three estimates is evaluated by their respective bias and their respective standard deviation of each component. The minimum reachable standard deviation given by the CRLB is also computed. All these quantities are presented in Table I for scenario 1 and in Table II for scenario 2.

For each case, three figures have been plotted: the first one depicts the set of PSLE; in the second figure, the set of the UPSLE is plotted; the third one shows the AMLE.

\section{$\underline{\text { Scenario } 1}$}

TABLE I

\begin{tabular}{|c||c|c|c||c|c|c|c|}
\hline \multicolumn{1}{|c||}{} & \multicolumn{3}{c||}{ Bias } & \multicolumn{4}{c|}{ Standard deviation } \\
\cline { 2 - 8 } & PSLE & UPSLE & AMLE & PSLE & UPSLE & AMLE & CRLB \\
\hline \hline $0(\mathrm{~m})$ & 14.8 & 4.1 & 6.4 & 20.6 & 23.9 & 20.6 & 19.2 \\
\hline $9000(\mathrm{~m})$ & -2396.1 & -471.4 & 177.4 & 1265.1 & 1616.9 & 1689.1 & 1707.7 \\
\hline $6(\mathrm{~m} / \mathrm{s})$ & -1.65 & -0.33 & 0.09 & 0.85 & 1.09 & 1.12 & 1.14 \\
\hline $0(\mathrm{~m} / \mathrm{s})$ & -0.02 & -0.01 & -0.01 & 0.47 & 0.54 & 0.42 & 0.38 \\
\hline $500(\mathrm{~Hz})$ & -0.111 & -0.023 & 0.006 & 0.176 & 0.201 & 0.166 & 0.153 \\
\hline
\end{tabular}

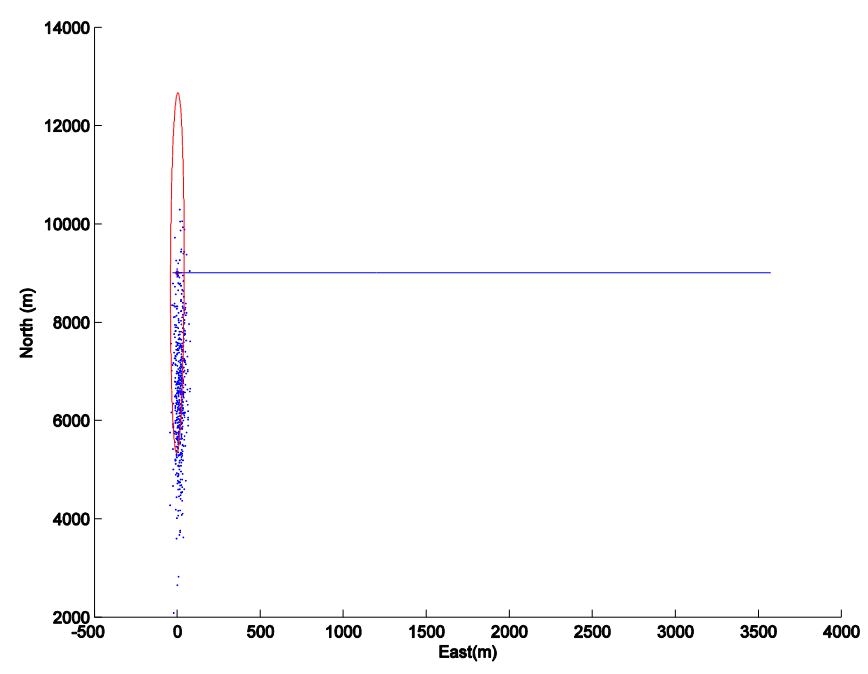

Fig. 1. Pseudolinear estimate in Scenario 1. 


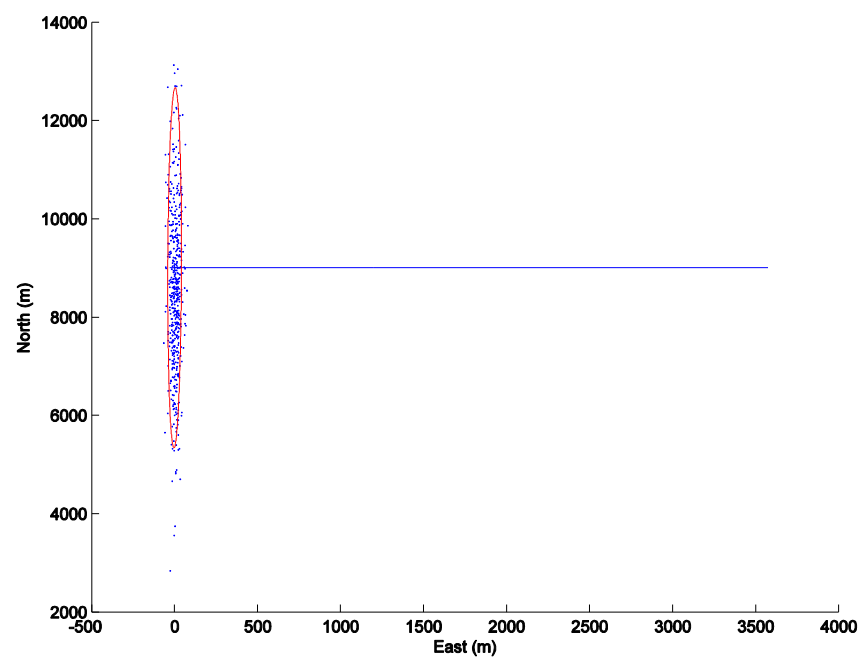

Fig. 2. Unbiased pseudolinear estimate for Scenario 1.

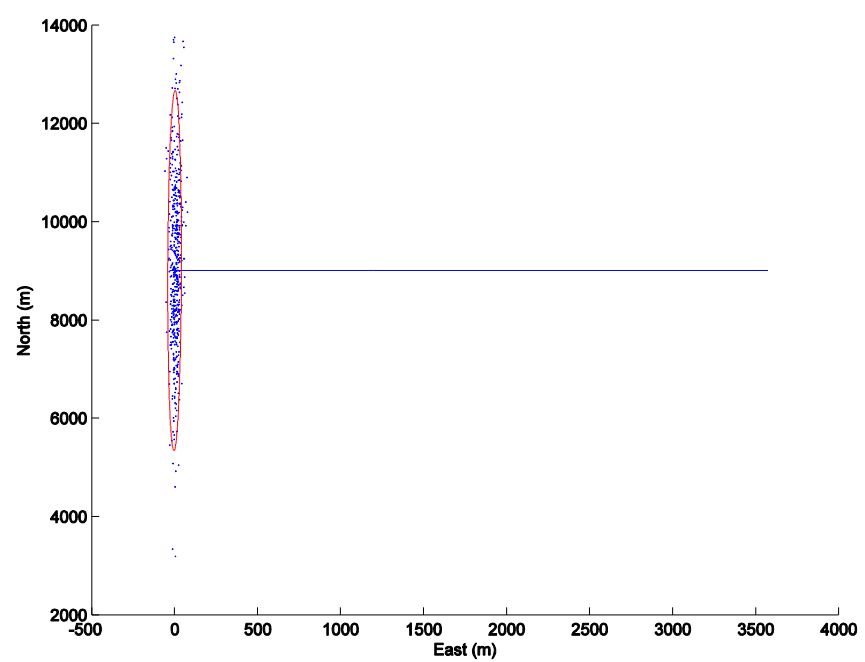

Fig. 3. Approximate maximum likelihood estimate for Scenario 1.

\section{Scenario 2}

TABLE II

\begin{tabular}{|c||c|c|c||c|c|c|c|}
\hline \multicolumn{1}{|c||}{$X$} & \multicolumn{4}{c||}{ Bias } & \multicolumn{4}{c|}{ Standard deviation } \\
\cline { 2 - 8 } & PSLE & UPSLE & AMLE & PSLE & UPSLE & AMLE & CRLB \\
\hline \hline$-3576(\mathrm{~m})$ & 1065.5 & 311.9 & 52.0 & 537.4 & 691.6 & 722.8 & 678.0 \\
\hline $9000(\mathrm{~m})$ & -2646.2 & -774.3 & -115.2 & 1357.7 & 1741.3 & 1825.8 & 1710.4 \\
\hline $6(\mathrm{~m} / \mathrm{s})$ & -1.81 & -0.53 & -0.10 & 0.90 & 1.16 & 1.21 & 1.14 \\
\hline $0(\mathrm{~m} / \mathrm{s})$ & 0.01 & -0.00 & 0.01 & 0.47 & 0.53 & 0.42 & 0.38 \\
\hline $500(\mathrm{~Hz})$ & 0.119 & 0.034 & 0.009 & 0.166 & 0.191 & 0.163 & 0.153 \\
\hline
\end{tabular}

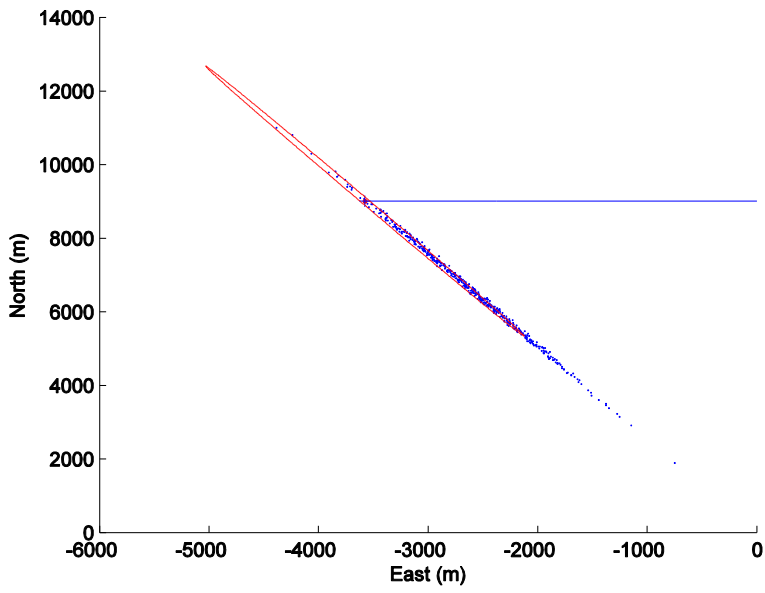

Fig. 4. Pseudolinear estimate in Scenario 2.

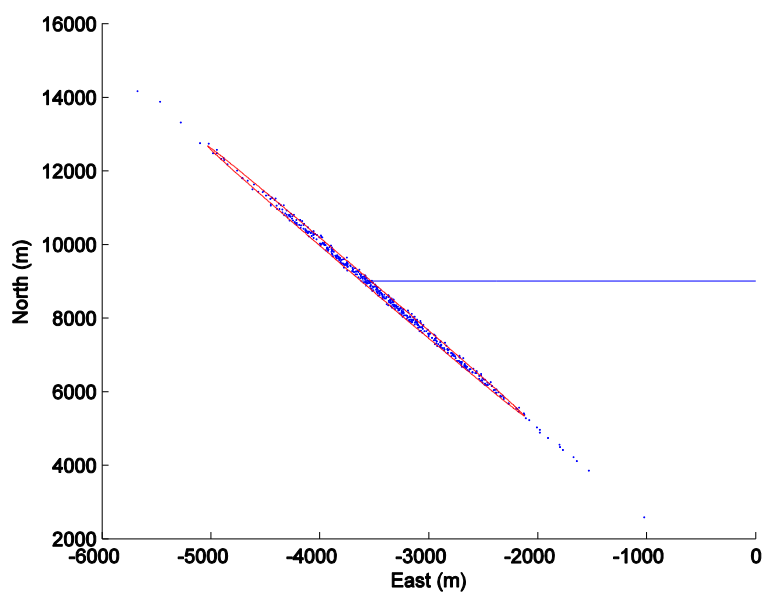

Fig. 5. Unbiased pseudolinear estimate for Scenario 2.

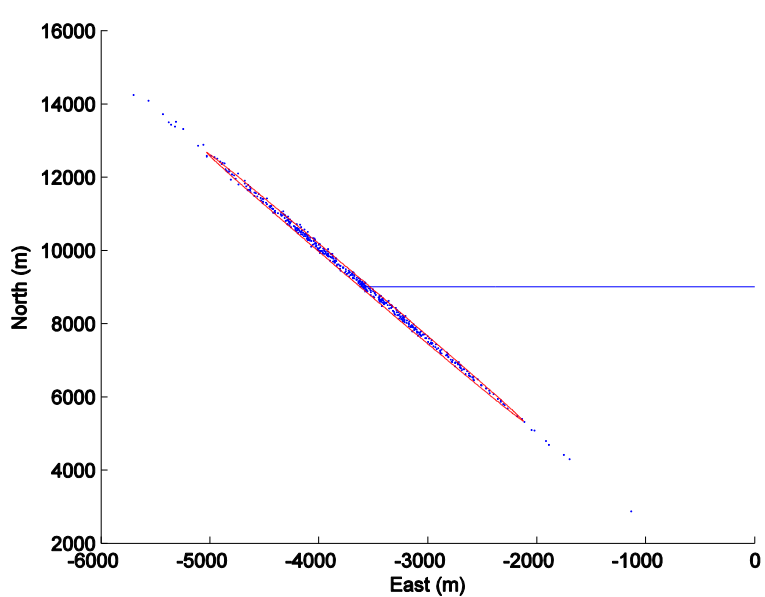

Fig. 6. Approximate maximum likelihood estimate for Scenario 2.

We can conclude that the two approaches to reduce the bias work properly and the obtained estimates (AMLE and UPSLE) are practically efficient. 


\section{CONCLUSION}

In this paper, we presented a proof of observability of a source (emitting a single tone) moving at constant velocity in a plane from a motionless observer which acquired simultaneously a measured bearing and a measured frequency.

We proposed to estimate the state vector by using a linear representation of the measurement, yielding to the so-called pseudolinear estimate. Based on the statistical assumptions commonly used, we proposed to estimate the bias of this estimate (which is by essence biased because of the presence of the bearing noise in the state matrix and in the measurements). Two ways were explored: one yielding the maximum likelihood estimate and the second based upon the method of moments. The estimated bias is removed. The two final estimates are practically efficient. This technique could be adapted to the case of multi-frequency and bearings TMA [13].

\section{APPENDIX}

\section{COMPUTATION OF THE COVARIANCE MATRIX OF THE NOISE}

If the random variable $Z$ has a normal (or Gaussian) distribution with parameter $\mu$ (mean) and $\sigma$ (standard deviation), then $\mathbf{E}(\cos a Z)=\cos (a \mu) \exp \left(-\frac{a^{2} \sigma^{2}}{2}\right)$.

If $\quad \mu=0, \quad$ then $\quad \mathbf{E}(\cos Z)=\exp \left(-\frac{\sigma^{2}}{2}\right)$ and

$\operatorname{Var}(\cos Z)=\frac{1}{2}+\frac{1}{2} \exp \left(-2 \sigma^{2}\right)-\exp \left(-\sigma^{2}\right)$.

We have also $\mathbf{E}(\sin Z)=0$ and $\operatorname{Var}(\sin Z)=\frac{1}{2}-\frac{1}{2} \exp \left(-2 \sigma^{2}\right)$

When $\sigma<<1$, then $\operatorname{Var}(\cos Z) \cong \frac{\sigma^{4}}{2}$. The random variable $\cos Z$ can be considered as the deterministic value equal to $\exp \left(-\frac{\sigma^{2}}{2}\right) \cong 1$. And $\operatorname{Var}(\sin Z) \cong \sigma^{2}$.

\section{Lemma}

Let us consider a deterministic quantity $\theta$ and $a$ Gaussian 0 -mean noise $\varepsilon$ of variance $\sigma^{2}$, then

$\mathbf{E}[\cos \varepsilon \sin \varepsilon]=0$

$$
\begin{aligned}
& \mathbf{E}[\cos (\theta+\varepsilon)]=\cos \theta \exp \left(-\frac{\sigma^{2}}{2}\right) \stackrel{\sigma<<1}{\cong} \cos \theta \\
& \mathbf{E}[\sin (\theta+\varepsilon)]=\sin \theta \exp \left(-\frac{\sigma^{2}}{2}\right) \stackrel{\sigma<<1}{\cong} \sin \theta \\
& \mathbf{E}\left[\cos ^{2}(\theta+\varepsilon)\right]=\frac{1+\cos 2 \theta \exp \left(-2 \sigma^{2}\right)}{2} \stackrel{\sigma<<1}{\cong} \cos ^{2} \theta \\
& \mathbf{E}\left[\sin ^{2}(\theta+\varepsilon)\right]=1-\mathbf{E}\left[\cos ^{2}(\theta+\varepsilon)\right]=\frac{1-\cos 2 \theta \exp \left(-2 \sigma^{2}\right)}{2} \\
& \quad \stackrel{\sigma<<1}{\cong} \sin ^{2} \theta \\
& \mathbf{E}[\cos (\theta+\varepsilon) \sin \varepsilon] \stackrel{\sigma<<1}{\cong}-\sigma^{2} \sin \theta,
\end{aligned}
$$

$\mathbf{E}[\sin (\theta+\varepsilon) \sin \varepsilon] \stackrel{\sigma<<1}{\cong} \sigma^{2} \cos \theta$.

Computation of the first two moments of $\xi_{k}$.

Recall that $\xi_{k}=\left[\begin{array}{c}-f_{0} r_{k} \sin \varepsilon_{k} \\ f_{0} \frac{v}{c} \sin \left(\theta_{k}-h\right) \sin \varepsilon_{k}-\eta_{k}\end{array}\right]$.

Obviously, $\mathbf{E}\left(\xi_{k}\right)=\left[\begin{array}{l}0 \\ 0\end{array}\right]$.

The statistical independence between $\varepsilon_{k}$ and $v_{k}$ allows us to simplify the expression of its covariance matrix:

$$
\operatorname{Cov}\left(\xi_{k}\right)=\mathbf{E}\left[\begin{array}{cc}
\gamma_{0}^{2} r_{k}^{2} \sin ^{2} \varepsilon_{k} & -\gamma_{0}^{2} r_{k} \frac{v}{c} \sin \left(\theta_{k}-h\right) \sin ^{2} \varepsilon_{k} \\
-\gamma_{0}^{2} r_{k} \frac{v}{c} \sin \left(\theta_{k}-h\right) \sin ^{2} \varepsilon_{k} & \gamma_{0}^{2} \frac{v^{2}}{c^{2}} \sin ^{2}\left(\theta_{k}-h\right) \sin ^{2} \varepsilon_{k}+\eta_{k}^{2}
\end{array}\right]
$$

Using the approximation,

$$
\begin{aligned}
\mathbf{E}\left(\sin ^{2} \varepsilon_{k}\right) & =\frac{1}{2}-\frac{1}{2} \mathbf{E}\left[\cos \left(2 \varepsilon_{k}\right)\right] \\
& =\frac{1}{2}-\frac{1}{2} \exp \left(-2 \sigma_{\theta}^{2}\right) \\
& \cong \sigma_{\theta}^{2},
\end{aligned}
$$

we get

$$
\operatorname{Cov}\left(\xi_{k}\right)=\left[\begin{array}{cc}
\gamma_{0}^{2} r_{k}^{2} \sigma_{\theta}^{2} & -\gamma_{0}^{2} r_{k} \frac{v}{c} \sin \left(\theta_{k}-h\right) \sigma_{\theta}^{2} \\
-\gamma_{0}^{2} r_{k} \frac{v}{c} \sin \left(\theta_{k}-h\right) \sigma_{\theta}^{2} & \gamma_{0}^{2} \frac{v^{2}}{c^{2}} \sin ^{2}\left(\theta_{k}-h\right) \sigma_{\theta}^{2}+\sigma_{f}^{2}
\end{array}\right]
$$

QED.

\section{REFERENCES}

[1] S.C. Nardone, A.G. Lindgren, and K.F. Gong, "Fundamental Properties and Performance of Conventional Bearings-Only Target Motion Analysis", IEEE Trans. Autom. Control, vol. 29, 9, pp. 775787, Sept. 1984.

[2] J.M. Passerieux, D. Pillon, P. Blanc-Benon and C. Jauffret, “ Target Motion Analysis with Bearings and Frequency Measurements via Instrumental Variable Estimator", in Proceedings of the International Conference on Acoustics, Speech, and Signal Processing, Glasgow, Scotland, May 1989.

[3] V.J. Aidala and S. Hammel, "Utilization of Modified Polar Coordinates for Bearings-Only Tracking", IEEE Trans. Autom. Control, vol. 28, no. 3, pp. 283-294, March 1983.

[4] D. Laneuville and C. Jauffret, "Recursive Bearings-Only TMA via Unscented Kalman Filter: Cartesian vs. Modified Polar Coordinates", IEEE Aerospace Conference, Big Sky, Montana, USA, March 2008.

[5] S. Arulampalam, B. Ristic, N. Gordon and T. Mansell, "Bearings-only Tracking of Manoeuvering Targets using Particle Filters", EURASIP Journal on Applied Signal Processing, vol. 15, pp. 2351-2365, January 2004.

[6] C. Jauffret, and D. Pillon, "Observability in Passive Target Motion Analysis", IEEE Trans. Aerosp. Electron. Syst., AES, vol. 32, no. 4, pp. 1290-1300, October 1996

[7] D.W. Whitcombe, "Pseudo-state Measurements Applied to Recursive Nonlinear Filtering", Proceedings of the 3rd Symposium on Estimation Theory and Its Application, San Diego, USA, pp. 278-281, 1972.

[8] K. Doğançay, "3D Pseudolinear target Motion Analysis from Angle Measurements", IEEE Trans. Signal Process., vol. 63, no. 6, pp. 15701580, March 2015. 
[9] Y.T. Chan and S.W. Rudnicki, "Bearings-Only and Doppler Bearings Tracking Using Instrumental Variable", IEEE Transactions on Aerospace and Electronic Systems, vol. 28, no. 4, pp. 1076-1082, October 1992.

[10] K.C. Ho, Y.T. Chan, "An Asymptotically Unbiased Estimator for Bearings-Only and Doppler-Bearing Target Motion Analysis", IEEE Trans. on SP, vol. 54, no. 3, pp. 809-822, March 2006.

[11] T. S. Ferguson, "A course in large sample theory”, Chapman \& Hall, 1996.
[12] S. Kay, "Fundamentals of Statistical Signal Processing: Estimation Theory", Prentice Hall Signal Processing Series, Englewood Cliffs, New Jersey, 1993.

[13] C. Jauffret, P. Blanc-Benon and D. Pillon, "Multi Frequencies And Bearing TMA: Properties and Sonar Applications", Proceedings of the International Conference on Information Fusion, Cologne, Germany, July 2008. 